\title{
UN ARTEFACTO EN ASTA DE HUEMUL (HIPPOCAMELUS BISULCUS) EN DEPÓSITOS ARQUEOLÓGICOS DE LA COSTA ATLÁNTICA. IMPLICACIONES PARA LA MOVILIDAD HUMANA Y LA DISTRIBUCIÓN DE LA ESPECIE.
}

\author{
ISABEL CRUZ, A. SEBASTIÁN MUÑOZ** Y M. SOLEDAD CARACOTCHE**
}

\begin{abstract}
RESUMEN
En este trabajo se discute la distribución pasada del huemul (Hippocamelus bisulcus), cérvido endémico de los bosques subantárticos patagónicos, así como su uso por poblaciones humanas del Holoceno. A partir de un resto hallado en la costa atlántica (Punta Entrada, Santa Cruz, Argentina) se evalúan las implicaciones que del mismo derivan respecto de estimaciones acerca de su distribución generadas a partir de información histórica. Asimismo, se discute su significado en el marco de los estudios de movilidad humana en el sur de Patagonia durante los últimos 1.500 años. Para ello se describe previamente el contexto de recuperación del artefacto y sus características morfológicas. Se concluye que si bien el artefacto recuperado en Punta Entrada no es evidencia suficiente para avalar el aprovechamiento de un animal cazado en la localidad, su presencia en la costa puede estar indicando la movilidad humana o la circulación de objetos a lo largo de grandes distancias.
\end{abstract}

PALABRAS CLAVES: Hippocamelus bisulcus, zooarqueología, movilidad humana, distribución histórica, Patagonia.

\section{A HUEMUL (HIPPOCAMELUS BISULCUS) ANTLER ARTEFACT IN ARCHAEOLOGICAL DEPOSITS OF THE ATLANTIC COAST. IMPLICATIONS FOR HUMAN MOBILITY AND SPECIES DISTRIBUTION}

\begin{abstract}
This paper discusses the past distribution of the huemul (Hippocamelus bisulcus), an endemic cervid in Subantarctic Patagonian forests, as well as its utilization by human populations. Based on a huemul specimen found on the Atlantic coast (Punta Entrada, Santa Cruz, Argentina), its implications are assessed regarding distributional estimations generated from historical information as well as regarding human mobility in southern Patagonia throughout the last 1500 years. Previously, the recovery context

* Unidad Académica Río Gallegos, Universidad Nacional de la Patagonia Austral. isabelcruz55@yahoo.com.ar. Argentina.

* Laboratorio de Zooarqueología y Tafonomía de Zonas Áridas. Conicet-Museo de Antropología, Universidad Nacional de Córdoba. Argentina. smunoz@conicet.gov.ar.

*ss Delegación Regional Patagonia, Administración de Parques Nacionales. Argentina. scaracotche@apn.gov.ar.
\end{abstract}


of the object and its morphological characteristics are described. The paper concludes that even though the specimen recovered at Punta Entrada is not enough evidence to suggest the utilization of an animal hunted nearby, its presence on the coast may be interpreted as an indication of human mobility or the circulation of goods throughout long distances.

KEYWORDS: Hippocamelus bisulcus, zooarchaeology, human mobility, historical distribution, Patagonia.

\section{INTRODUCCIÓN}

... y hallaron una punta de un cuerno de çiervo, ques señal que hay tales animales en aquella tierra (Fernández de Oviedo y Valdés 1944:216).

Durante los trabajos de campo efectuados en noviembre de 2006 en Punta Entrada (Santa Cruz, Argentina) recuperamos un artefacto confeccionado en asta de huemul (Hippocamelus bisulcus) en un contexto de conchero a unas decenas de metros de la costa marina actual. La cita de Fernández de Oviedo hace referencia a un hallazgo similar, efectuado en el estrecho de Magallanes por integrantes de la expedición de García Jofré de Loaysa durante el siglo XVI. La interpretación que hacen los expedicionarios es simple: la presencia del asta remite directamente a la existencia de cérvidos en la localidad. Desde el punto de vista de la arqueología, sin embargo, las inferencias posibles son varias y el hallazgo de Punta Entrada no puede ser interpretado de manera tan directa.

El huemul es un cérvido endémico de los bosques subantárticos de la Argentina y Chile (Cabrera 1957; Serret 2001). Es el más robusto de los ciervos autóctonos del sur de América, pudiendo alcanzar hasta $100 \mathrm{~kg}$ de peso. El rango de distribución del huemul se extiende a lo largo de los Andes patagónicos, en el bosque y las áreas costeras del sur del océano Pacífico. Actualmente, su presencia no ha sido detectada en hábitats de estepa y sólo hay unos pocos registros en sitios ecotonales con el bosque andino-patagónico en Argentina (Vila et al. 2006). En Chile, la distribución actual del huemul abarca

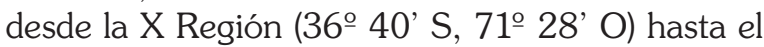

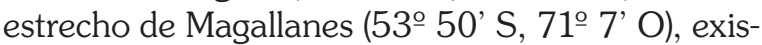
tiendo un núcleo aislado en Chillán (VIII Región). En la Argentina, el límite norte de la distribución de esta especie estaría en la provincia de Neuquén (40ํㅇํ $\mathrm{S}, 71^{\circ} 49^{\prime} \mathrm{O}$ ), mientras que el sur se ubica

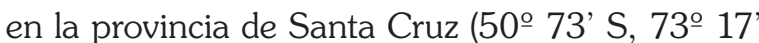
O) (Administración de Parques Nacionales 2007). El registro más oriental de la distribución corresponde a la provincia de Río Negro (41ํㅡㄴ 35' 3, $71^{\circ} 11^{\prime}$ 49" O) (Vila et al. 2006).

En función de la distribución actual del huemul, la presencia de este artefacto en el litoral atlántico sólo puede ser explicada en relación a la movilidad de objetos o de personas. Esto implica que, de alguna manera, las poblaciones humanas de la costa tenían contacto con la zona de bosque en la cordillera. Sin embargo, se ha propuesto que la distribución pasada del huemul abarcaba un área más extensa, incluyendo sectores de la costa atlántica (Díaz 1990, 1993, 2000; Vila et al. 2005). Si este fue el caso, entonces la presencia de este artefacto podría entenderse como la utilización de recursos de origen local.

En Patagonia se han recuperado huesos de huemul en registros arqueológicos de la zona cercana a la cordillera de los Andes, en contextos de bosque o en sectores aledaños del ecotono y la estepa. Debido a la escasez de animales de gran tamaño en el bosque, el huemul debió ser una de las presas elegidas por los cazadores humanos en estos ambientes, tanto en Argentina como en Chile (Belardi y Gómez Otero 1998; De Nigris 2004; Díaz et al. 2007; Fernández 2006; Mena 1992; Mena y Jackson 1991; Mena et al. 2004; entre otros).

Las menciones de huemul en la costa atlántica no han sido corroboradas hasta el momento por el registro arqueológico, que no ha brindado información que permita establecer la presencia de este cérvido en el litoral durante algún momento del Holoceno. El objetivo de este trabajo, por lo tanto, es presentar este artefacto confeccionado en asta de huemul, evaluar su aporte para los estudios sobre la movilidad de las poblaciones humanas de la zona y discutir las estimaciones de la distribución pasada de la especie derivadas del registro histórico. 


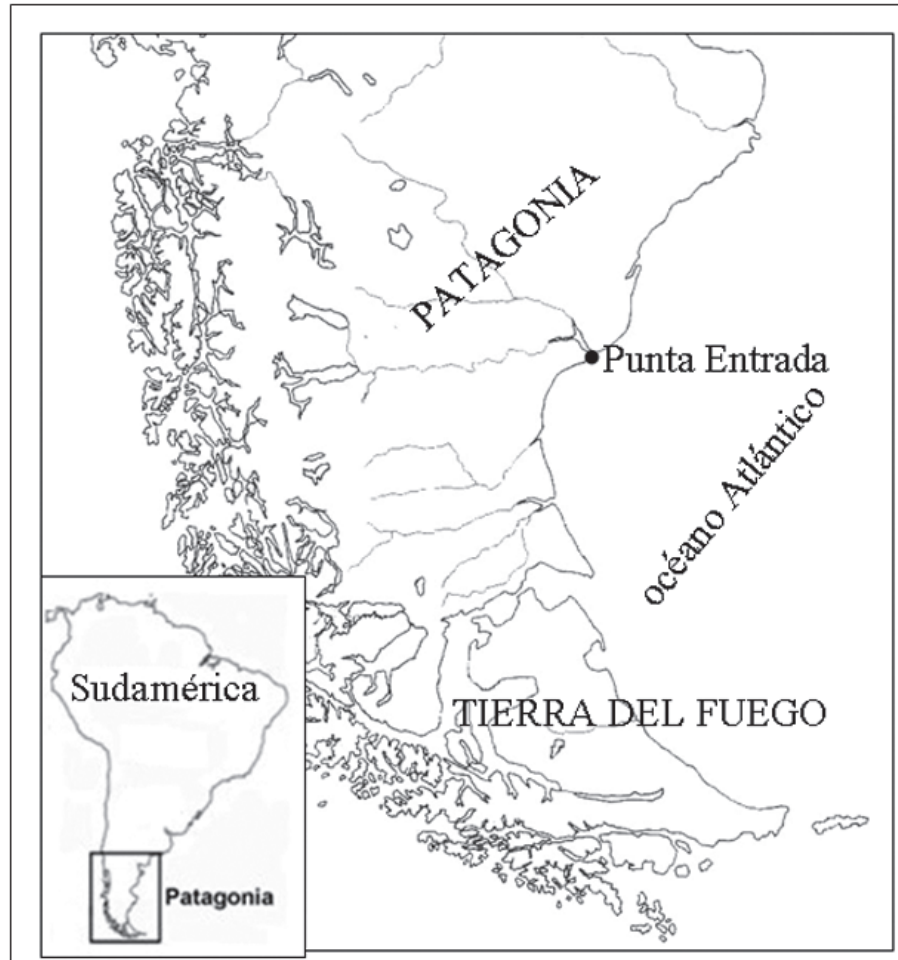

a)

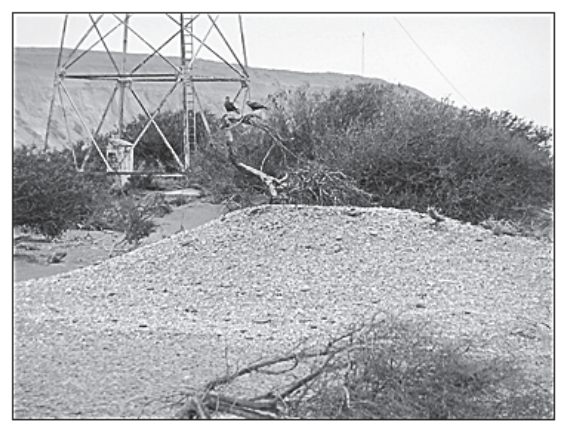

b)

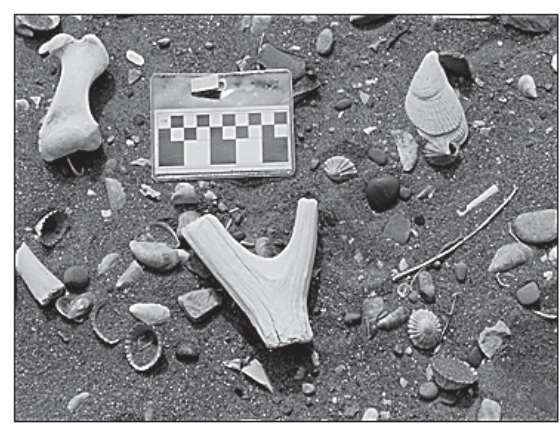

c)

Fig. 1. El contexto de recuperación del artefacto en asta de huemul (Hippocamelus bisulcus).

a) Ubicación de Punta Entrada (Santa Cruz, Argentina); b) Vista general del Punto 35; c) El artefacto en asta de huemul (H. bisulcus).

\section{EL ARTEFACTO Y SU CONTEXTO DE RECUPERACIÓN}

Punta Entrada se ubica en la margen sur de la desembocadura del río Santa Cruz (50ㅜ 08'S 682'ㅇ), en la provincia de Santa Cruz, Argentina (Fig. 1a). El registro arqueológico de Punta Entrada se caracteriza por la presencia de depósitos de tipo conchero a cielo abierto. En uno de los concheros ubicados en el área de médanos, el denominado Punto 35 (Fig. 1b), se recuperó el artefacto confeccionado en asta de huemul. Aunque afectado por diversos procesos naturales, este conchero presenta un cierto grado de integridad en el sector central del depósito. Las primeras dataciones obtenidas para este depósito se presentan en la Tabla 1, entre ellas un fechado radiocarbónico por AMS efectuado directamente sobre el asta.

El artefacto fue localizado en el sector afectado por la erosión, en superficie y asociado a otros materiales arqueológicos (Fig. 1c). Al momento de su recuperación presentaba un buen estado general de preservación. Su color es blanco grisáceo, con manchas más oscuras en una de sus caras. Además, posee grietas paralelas a la estructura de la fibra en ambas caras, que pueden atribuirse a la meteorización. El asta fue modificada con la técnica de marcado perimetral (sensu Muñoz y Belardi 1998), que en

Tabla 1. Fechados radiocarbónicos del Punto 35 (Punta Entrada, Santa Cruz, Argentina).

\begin{tabular}{|l|c|c|c|c|}
\hline Material & $\begin{array}{c}\text { Fechados radiocarbónicos } \\
\text { (Años calibrados AP.) }\end{array}$ & $\begin{array}{c}\text { Calibración por } \\
\text { efecto reservorio }\end{array}$ & $\delta 15 \mathrm{~N}(\% 0)$ & $\delta 13 \mathrm{C}(\% 0)$ \\
\hline Asta de huemul & $1.150 \pm 70$ (UGAMS 02946) & - & $+0,34$ & $-21,02$ \\
\hline Valvas & $970 \pm 50$ (LP-1844) & $570 \pm 50 \mathrm{AP}$ & - & - \\
\hline Hueso pinnípedo & $1.710 \pm 70$ (LP-1791) & $1.310 \pm 70 \mathrm{AP}$ & - & - \\
\hline
\end{tabular}




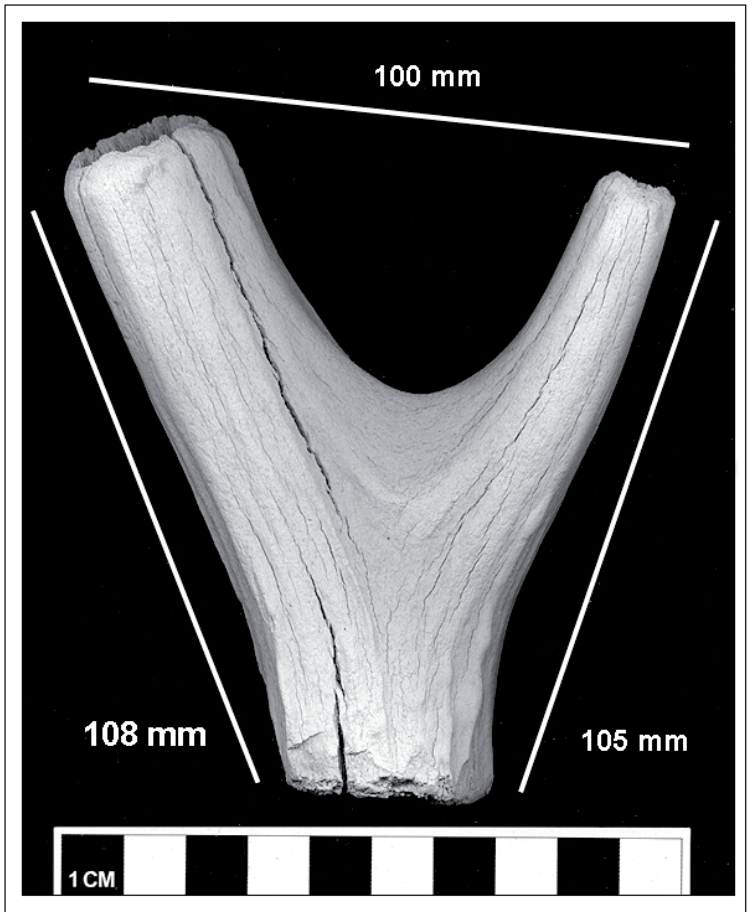

Fig. 2. Dimensiones del artefacto en asta de huemul recuperado en Punta Entrada (Santa Cruz, Argentina).

otros casos arqueológicos ha sido considerada como una manera eficiente de superar la resistencia de la dura capa compacta característica de este material (ver van Gijn 2007). La aplicación de esta técnica permitió seccionar los extremos de las dos ramas y la roseta (o base) del asta. De esta manera, quedó configurado un segmento con tres extremos abiertos y forma de horquilla, tal como puede apreciarse en la Fig. 2.

La presencia de restos de astas de cérvidos en el registro arqueológico puede vincularse con distintas condiciones de obtención, determinadas por el objetivo a partir del cual fueron procuradas: primaria, si la recolección es efectuada con para obtener materia prima, o secundaria, si proceden de la utilización de las astas de un animal cazado para alimentación (Vercouterè et al. 2007). Aunque los dos modos han sido postulados para el aprovechamiento de astas de huemul en Patagonia (Fernández 2006), no es posible determinar el tipo de obtención de la recuperada en Punta Entrada. Lo que puede afirmarse es que en casos como el que aquí analizamos, donde no existen registros actuales ni arqueológicos que confirmen la presencia de cérvidos en la costa, tanto el transporte del asta desde la zona cordillerana como su obtención por intercambio para la utilización como materia prima son altamente plausibles, particularmente si se tiene en cuenta que las astas se degradan rápidamente (van Gijn 2007).

Este artefacto puede ser tanto un instrumento como un desecho de manufactura. Scheinsohn y Lucero (2006) reportan un instrumento confeccionado con el extremo de un asta de huemul en la capa 11 del sitio CCP 7 (P. N. Perito Moreno, Santa Cruz, Argentina), fechada en 7.880 años AP. El artefacto de CCP 7 es caracterizado por estas autoras como una punta roma o retocador. Es posible que el fragmento de asta recuperado en Punta Entrada sea el desecho derivado de la confección de instrumentos de este tipo. El análisis de la pieza pone en evidencia que los segmentos más valorados desde el punto de vista tecnológico (la roseta y los extremos del asta) están ausentes. Además, no se han detectado evidencias claras de pulido por uso en los extremos (para ejemplos al respecto, ver van Gijn 2007: Fig. 8), que muestran cortes y lascados vinculados con la fractura dirigida de la pieza. Ambas características sustentan la determinación como desecho.

Por otro lado, también se ha propuesto que el marcado perimetral es el resultado del uso del hueso como instrumento de percusión o machacado (Hadjuk y Lezcano 2005), lo cual podría extenderse a las astas. En Punta Entrada se han registrado numerosos casos de presencia de esta técnica, tanto en huesos largos de guanaco como de pinnípedos y ñandúes (Caracotche, Cruz y Muñoz, observaciones personales). Dadas las dificultades para obtener astas en la costa, no pensamos que la misma fuera recolectada, transportada o intercambiada con el fin exclusivo de usarla como machacador. Sin embargo, no es posible descartar que, luego de aprovechar algunos segmentos para la confección de instrumentos, el desecho fuera utilizado de esta manera.

En síntesis, consideramos que el artefacto en asta de huemul recuperado en Punta Entrada es el subproducto del procesamiento para la obtención de los segmentos mas regulares del asta con el fin de confeccionar instrumentos y que es posible que el desecho derivado de la manufactura fuese utilizado luego para otras funciones, entre ellas la de machacador. 


\section{ESTIMACIONES DE LA DISTRIBUCIÓN HISTÓRICA DEL HUEMUL}

La evaluación de la distribución histórica del huemul ha sido abordada por Díaz (1990, 1993, 2000) utilizando información proveniente de narraciones de exploradores, viajeros y observadores de fauna. Según esta autora, varias de las fuentes desde el siglo XVI mencionan el avistaje de venados en dos puertos de la costa atlántica de Patagonia: Puerto Deseado y Puerto San Julián, así como en el área comprendida entre el río Santa Cruz y el estrecho de Magallanes. Propone dos hipótesis relativas a la distribución del huemul: a) este cérvido originalmente habitó toda la Patagonia y su retracción hacia la zona andina sería consecuencia de su vulnerabilidad frente a los cambios ecológicos y a la aparición de asentamientos humanos desde el siglo XIX; y b) la especie vivió en los ambientes boscosos andinos y sólo ocasionalmente alcanzó la costa atlántica.

Vila y colaboradores (2005) también evalúan las posibles variaciones en la distribución de este cérvido utilizando, además de referencias históricas, información arqueológica, relatos de antiguos pobladores, toponimia, localidades de distribución actual y avistajes pasados. Su objetivo es definir distintos escenarios de distribución histórica para la especie, con el fin de estimar su reducción potencial en el presente. Consideran que en el pasado histórico el hábitat del huemul no sólo abarcaba las zonas boscosas del área cordillerana, sino que también incluía el ecotono e incluso sectores de estepa hasta la costa atlántica. Coinciden con Díaz en que fueron posibles las ingresiones hasta el litoral atlántico a través de cuencas hidrográficas y áreas quebradas, lo que ya había sido sugerido por otros autores a comienzos del siglo XX. Sin embargo, aclaran que los escenarios que proponen podrían estar sobreestimando ampliamente la distribución de la especie (Vila et al. 2005).

\section{EL ARTEFACTO COMO INDICADOR DE MOVILIDAD HUMANA}

En Patagonia, los estudios arqueológicos se han centrado en algunos de los indicadores de movilidad y circulación de objetos, especialmente las materias primas líticas con que se confeccionaron algunos artefactos y los análisis de isótopos estables en huesos humanos. Los restos de fauna incluidos en los depósitos arqueológicos también pueden ser indicadores interesantes de movilidad y circulación. La presencia de valvas de moluscos o restos de vertebrados marinos en depósitos alejados de la costa (Borrero 2001; Barberena y Borrero 2006; entre otros), así como las plumas y huesos de ciertas aves en contextos que no se corresponden con el hábitat o el área de distribución de las mismas (Humphrey et al. 1993; entre otros), han sido utilizados para establecer el rango de acción de las poblaciones humanas y el uso de ciertos ambientes de la región.

El artefacto confeccionado en asta de huemul recuperado en Punta Entrada puede ser considerado desde este punto de vista. Si se acepta que la distribución pasada del huemul se restringía al área de bosque y ecotono, tal como sucede en la actualidad, entonces la presencia de este artefacto nos permite inferir algún tipo de relación entre la costa marina y la cordillera, distante aproximadamente unos $250 \mathrm{~km}$ hacia el Oeste. Esta relación puede haber estado dada por la movilidad de las poblaciones humanas desde Punta Entrada en la costa marina hasta el área de bosque y ecotono cercana al lago Argentino, posiblemente a lo largo del valle del río Santa Cruz, o a través del río Chico hasta el área de los lagos Belgrano y Nansen -en donde actualmente habitan huemules (Serret 2001)-. También podría indicar el desplazamiento de poblaciones desde o hacia el estrecho de Magallanes y sus alrededores, en donde se ha recuperado evidencia arqueológica que señala la utilización de este cérvido (Belardi y Gómez Otero 1998; Díaz et al. 2007; entre otros). De la misma manera, puede indicar la circulación de bienes entre poblaciones ubicadas en los diferentes ambientes mencionados.

\section{DISCUSIÓN}

Las fuentes históricas y el registro arqueológico pueden ser esenciales para discutir diversos aspectos de la historia natural de la fauna y los paisajes (Lyman y Cannon 2004; entre otros). Sin embargo, un uso correcto de cualquiera de estos registros implica conocer sus límites y alcances para aportar al tema en cuestión. Uno de los problemas de las inferencias basadas en las referencias históricas tiene que ver con los términos equívocos o que han variado su significado a lo largo del tiempo. En su discusión acerca de la distribución histórica del huemul, Díaz 
(1993) plantea que las referencias al huemul en las fuentes históricas (especialmente las más tempranas) deben tomarse con cautela ya que este animal era nuevo para los europeos, que por lo tanto carecían de habilidad para identificarlo y describirlo. Debido a ello, excluye todas aquellas menciones a ciervos o venados en las áreas en que podrían superponerse las distribuciones de huemules y venados de las pampas (Ozotoceros bezoarticus). Además, no considera las menciones del "anta", debido a que son poco claras y a que no necesariamente indican al huemul. Esta autora señala que Falkner describe al anta como una especie de venado sin cuernos y del tamaño de un asno grande (Díaz 1993). Tanto esta como otras descripciones ambiguas no pueden ser consideradas al postular la distribución pasada del huemul.

En este sentido, otro término que puede prestarse a confusiones es "venado". Según la edición del siglo XVIII del Diccionario de la Real Academia Española (Real Academia Española 1780), el término venado alude a una especie de ciervo del tamaño de un caballo, pero también se llamaba venado a cualquier res de caza mayor. Esto implica, por lo tanto, que las referencias a venados en las fuentes más tempranas también son ambiguas, ya que podrían hacer referencia tanto al huemul como a cualquier otro animal de porte semejante.

Las fuentes documentales de los primeros siglos de la exploración europea no siempre pueden ser consideradas como evidencia para discutir la distribución de una especie, debido principalmente a que las observaciones que incluyen no son sistemáticas y a que las descripciones tienen las limitaciones de la época. Su utilidad radica, en cambio, en que permiten visualizar situaciones diferentes a la actual $y$, a partir de ellas, formular hipótesis que luego podrán ser contrastadas con la evidencia derivada de otros registros -por ejemplo, el arqueológico-.

A diferencia de lo que ocurre en la región de Magallanes (Chile), en donde el registro arqueológico muestra la utilización del huemul en ambientes costeros (Díaz et al. 2007; entre otros), las hipótesis que plantean la presencia de la especie en sectores de la costa atlántica (Díaz 1993, 2000) no han sido corroboradas por el registro arqueológico. En este sentido, el enfoque más apropiado para contrastar estas hipótesis sería aquel que considere varias líneas de evidencia, entre ellas la biología y ecología actuales de la especie. Tanto la anatomía como la estructura corporal del huemul parecen seguir las adaptaciones observadas en otros ungulados de montaña del hemisferio Norte (Geist 1998), las cuales posiblemente sean respuestas evolutivas al uso de hábitat de las especies adaptadas a pendientes escarpadas (Frid 1994; entre otros). Por otro lado, estudios recientes en Chile han mostrado que los huemules adultos tienen rangos de acción pequeños y estables y que la mayoría de los individuos no efectúan movimientos a grandes distancias, sino sólo pequeños movimientos estacionales (Saucedo y Gill 2004). Además, los estudios de selección de hábitat del huemul efectuados por estos autores indican que evita las zonas de gramíneas y estepas. Todas estas características parecen incompatibles con la consideración de individuos de la especie desplazándose cientos de kilómetros a lo largo de planicies y zonas quebradas hasta sectores costeros en los que predominan las estepas y pastizales.

El artefacto recuperado en Punta Entrada tampoco sustenta estas hipótesis, ya que no es evidencia suficiente para avalar el aprovechamiento de un animal cazado en la localidad. Para que este fuera el caso, debería contarse con otros restos de huemul en los depósitos arqueológicos -especialmente huesos- y hasta el momento no se han reportado hallazgos de este tipo ni en Punta Entrada ni en otras localidades costeras del sur de Patagonia. En cambio, puede interpretarse como un objeto transportado por las poblaciones humanas desde el bosque o su ecotono con la estepa hasta la costa del mar, o como un bien que circuló de alguna manera entre poblaciones de estos ambientes a lo largo de grandes distancias. Esta es la manera más parsimoniosa de entender la presencia de este artefacto en el conchero de Punta Entrada, por lo menos hasta contar con evidencia que permita considerar otras opciones.

Los estudios de movilidad humana también requieren de la utilización de diversas líneas de evidencia. Los análisis de isótopos estables efectuados en restos humanos recuperados tanto en Punta Entrada como en Rincón del Buque -una localidad cercana- muestran dietas con componentes terrestres y marinos (Suby y Guichón 2007). El rango de acción de las poblaciones humanas de la región durante el Holoceno Tardío definido a partir de este indicador incluiría sectores de costa y del interior distante varios cientos de kilómetros, lo cual es concordante con lo planteado en relación al artefacto en asta. Lo 
mismo sucede con los artefactos confeccionados en obsidiana negra recuperados en Punta Entrada o en obsidiana negra y verde en Monte León (Caracotche et al. 2005), localidad ubicada inmediatamente al sur de Punta Entrada. En síntesis, todos estos indicadores estarían señalando que las poblaciones humanas del Holoceno Tardío en este sector del sur de Patagonia tenían rangos de acción amplios, que incluyeron los diversos ambientes de la región.

\section{CONCLUSIONES}

Tanto las fuentes históricas como el registro arqueológico han brindado elementos para discutir la distribución pasada del huemul, especialmente en la zona de bosque y ecotono a ambos lados de la cordillera, en el sector de fiordos e islas del sur de Chile y en el estrecho de Magallanes y zonas aledañas. Sin embargo, no existe concordancia entre ambos registros en la costa atlántica. Las referencias históricas son bastante escasas y confusas y, por el momento, el registro arqueológico no ha brindado elementos que avalen la presencia de la especie en este sector de la costa. El hallazgo del artefacto en asta de huemul recuperado en Punta Entrada no aporta elementos que permitan corroborar alguna de las hipótesis propuestas al respecto. En función de las hipótesis planteadas a partir de las fuentes históricas (Díaz 1993, Díaz 2000) y las consideraciones que hemos presentado a lo largo de este trabajo, pensamos que los análisis de restos de fauna en contextos arqueológicos de la costa atlántica serán los que tendrán un papel crucial en las discusiones de los próximos años en relación a los dos temas aquí tratados: distribución pasada del huemul y movilidad humana en función de este indicador.

\section{AGRADECIMIENTOS}

A Pablo Fernández, Eduardo Ramilo y Hernán Pastore por sus aportes, comentarios y sugerencias. A Claudia Aguilar, Carolina Moreno y Lorena Martínez, que colaboraron en las tareas de campo. A la Municipalidad de la ciudad de Puerto Santa Cruz, que brindó transporte y alojamiento durante los trabajos de campo. Al Sr. Víctor López de Estancia Monte Entrance. Este trabajo es un resultado de los Proyectos PIP/CONICET 5576, PIP/CONICET 112-200801-00996y UBACyT F 447.

\section{BIBLIOGRAFÍA}

ADMINISTRACIÓN DE PARQUES NACIONALES 2007. Programa de Conservación del Huemul, Argentina.

BELARDI, J. B. y J. GÓMEZ-OTERO 1998. Anatomía económica del huemul (Hippocamelus bisulcus): una contribución a la interpretación de las evidencias arqueológicas de su aprovechamiento en Patagonia. Anales del Instituto de la Patagonia, Serie Ciencias Humanas 26:195-207.

BORRERO, L. A. 2001. El poblamiento de la Patagonia. Toldos, milodones y volcanes. Emecé Editores, Buenos Aires, Argentina.

BORRERO, L. A. y R. BARBERENA 2006. Hunter-Gatherer Home Ranges and Marine Resources. An Archaeological Case from Southern Patagonia. Current Anthropology 47:855-867.

CABRERA, A. 1957. Catálogo de los mamíferos de América del Sur. Revista del Museo Argentino de Ciencias Naturales "B. Rivadavia" IV: 732.

CARACOTCHE, M. S.; I. CRUZ; S. ESPINOSA; F. CARBALLO Y J. B. BELARDI 2005. Rescate arqueológico en el Parque Nacional Monte León (Santa Cruz, Argentina). Magallania 33 (2):143-163.

DE NIGRIS, M. 2004. El consumo en grupos cazadores recolectores. Un ejemplo zooarqueológico de Patagonia Meridional. Sociedad Argentina de Antropología, Buenos Aires.

DÍAZ, N. I. 1990. El huemul: Antecedentes históricos. Edipubli S. A., Buenos Aires, Argentina.

DÍAZ, N. I. 1993. Changes in the range distribution of Hippocamelus bisulcus in Patagonia. Zeitschrift für Säugetierkunde 58:344-351.

DÍAZ, N. I. 2000. El huemul (Hippocamelus bisulcus Molina, 1782): Una perspectiva histórica. En: Díaz, N. I. y JM Smith-Flueck (editores) El huemul Patagónico, un misterioso cérvido al borde de la extinción: 1-32. LOLA, Buenos Aires, Argentina.

DÍAZ, N. I., A. PRIETO y G. BAHAMONDE 2007. Guanacos tímidos, huemules confiados: el límite occidental de los cazadores terrestres australes. Magallania 35(1):133-138.

FERNANDEZ, P. M. 2006. Aprovechamiento de recursos faunísticos en los ambientes de estepa y ecotono bosque-estepa del norte de la Provincia del Chubut. Tesis Doctoral, Facultad de Filosofía y Letras, Universidad de Buenos Aires, Argentina.

FERNÁNDEZ DE OVIEDO y VALDÉS, G. 1944. Historia general y natural de las Indias. Editorial Guarania, Asunción del Paraguay. 
FRID, A. 1994, Observations on habitat use and social organization of a huemul (Hippocamelus bisulcus) coastal population in Chile. Biological Conservation 67.

GEIST, V. 1998. Deer of the World. Their Evolution, Behavior and Ecology. Stackpole Books, USA.

HAJDUK A. y M. J. LEZCANO. 2005. Un "nuevo-viejo" integrante del elenco de instrumentos óseos de Patagonia: los machacadores óseos. Magallania 33 (1):63-80 63.

HUMPHREY, P. S., J. E. PEFAUR y P. C. RASMUSSEN. 1993. Avifauna of Three Holocene Cave Deposits in Southern Chile. University of Kansas Museum of Natural History Occasional Papers 154:1-37.

LYMAN, R. L. y K. P. CANNON. 2004. Zooarchaeology and conservation biology. The University of Utah Press, Salt Lake City, USA.

MENA, F. 1992. Mandíbulas y maxilares: un primer acercamiento a los conjuntos arqueofaunísticos del Alero Fontana (RI-22; XI Región). Boletín del Museo Nacional de Historia Natural de Chile 43: 179-191.

MENA, F. y D. JACKSON. 1991. Tecnología y subsistencia en Alero Entrada Baker, Región de Aysén, Chile. Anales del Instituto de la Patagonia, Serie Ciencias Sociales 20: 169-203.

MENA, F., H. VELÁSQUEZ, V. TREJO y J. C. TORRES MURA. 2004. Aproximaciones zooarqueológicas al pasado de Aisén continental (Patagonia central chilena). En: G. L. Mengoni Goñalons (editor) Zooarchaeology of South America, pp. 99-121. BAR Internacional Series 1298, Oxford, UK.

MUÑOZ, A. S. y J. B. BELARDI. 1998. El marcado perimetral en los huesos largos de guanaco de Cañadón Leona (Colección Junius Bird): implicaciones arqueofaunísticas para Patagonia Meridional. Anales del Instituto de la Patagonia, Serie Ciencias Sociales 26: 107-117.

REAL ACADEMIA ESPAÑOLA. 1780. Diccionario de la Lengua Castellana, Tomo sexto, pp. 439, Imprenta de la Real Academia Española, por los herederos de Francisco del Hierro, Madrid.
SAUCEDO, C. y R. GILL. 2004. Huemul (Hippocamelus bisulcus) ecology research: conservation planning in Chilean Patagonia. Deer Specialist Group News 19:13-15.

SCHEINSOHN, V. y M. LUCERO. 2006. Bone Raw Material Exploitation at the South of South America: The Cerro Casa de Piedra locality. Trabajo presentado en el VI ICAZ (Internacional Council of Archaeozoology) International Meeting, Ciudad de México (México), agosto 2006.

SERRET, A. 2001. Huemul. En: C. M, Dellafiore y N. Maceira (editores) Los ciervos autóctonos de la Argentina y la acción del hombre, pp. 57-66. Secretaría de Desarrollo Sustentable y Política Ambiental, Ministerio de Desarrollo Social y Medio Ambiente, Argentina.

SUBY, J. A. y R. A. GUICHÓN. 2007. Análisis de restos bioarqueológicos de la costa meridional de Santa Cruz. Trabajo presentado en las VI Jornadas de Antropología Biológica. Salta, octubre de 2007.

VAN GIJN, A. 2007. The use of bone and antler tools: two examples from the late Mesolithic in the Dutch Coastal Zone. En: C. Gates St-Pierre y R.B. Walker (editores) Bones as Tools: Current Methods and Interpretations in Worked Bone Studies, BAR S1622, pp. 81-92. Archaeopress, Oxford.

VERCOUTERÈ, C., M. PATOU-MATHIS y G. GIACOBINI. 2007. The importance of the paleontological and taphonomical analyses for the study of bone industries. En: C. Gates St-Pierre y R.B. Walker (editores) Bones as Tools: Current Methods and Interpretations in Worked Bone Studies, BAR S1622, pp. 23-34. Archaeopress, Oxford.

VILA, A. R., H. PASTORE, N. I. DÍAZ, R. LÓPEZ y R. FAÚNDEZ. 2005. Distribución pasada y actual del huemul: Escenarios de cambio potencial. Publicación técnica de WCS, CODEFF y FVSA. S. C. de Bariloche, Argentina y Concepción, Chile.

VILA, A. R., R LÓPEZ, H. PASTORE, R. FAÚNDEZ y A. SERRET. 2006. Current distribution and conservation of the huemul (Hippocamelus bisulcus) in Argentina and Chile. Mastozoología Neotropical 13:263-269. 